\section{Pathophysiology Haemostasis and Thrombosis}

For the Author Index of No. 5-6, please see pp. 409-410
Abdel-Raheem, M.M. 107

Aguirrebeitia, M.J. 115

Anderson, D.A. 131

Angelini, A. 180

Atar, D. 8

Barbera, N. 127

Bartels, P. 137

Basu, S. 85

Benattar, N. 194

Bergmann, J.-F. 134

Bermejo, E.I. 155

Bianchini, P. 44

Binz, C. 16

Biron-Andréani, C. 194

Bjerregaard Larsen, T. 92

Boekel, E. ten 137

Börretzen, J. 59

Brandjes, D.P.M. 76

Brandslund, I. 92

Braun, S. 16

Büller, H.R. 76, 111

Carmichael, A.R. 40

Casais, P. 155

Cate, H. ten 195

Caulin, C. 134

Chan, W.S. 51

Chassany, O. 134

Chung, N.A.Y. 158

Cincotta, M. 127

Cuccurullo, F. 180

Danielson, B.D. 107

De La Cruz, J.P. 25

Di Febbo, C. 180

Di Ilio, C. 180

Dönges, R. 143

Drouet, L. 134

Eguchi, Y. 165

Eldor, A. 99

Fahlén, M. 59

Fenyvesi, T. 174

Fraiman, G. 107

Fredholm, L. 92

Frisina, N. 127

Fujimura, Y. 33

Fuzaylov, S.Y. 8
Gang, Z. 165

Ganti, A.K. 80

Gattis, W.A. 8

Gaudio, A. 127

Gawaz, M. 16

Giddings, J.C. 184

González-Correa, J.A. 25

Grenard, A.-S. 134

Grönlund Thomsen, H. 59

Guerrero, A. 25

Gurbel, P.A. 8

Hanekom, D. 107

Harenberg, J. 174

Hasselblom, S. 59

Hayashi, T. 33

Heijden, J.F. van der 111

Hemker, H.C. 1

Hey, H. 92

Hoffmann, J.N. 143

Inthorn, D. 143

Iriarte, J.A. 115

Jochum, M. 143

Johnston, D. 40

Jörg, I. 174

Jung, F. 121

Kamphuisen, P.W. 190

Kapsoritakis, A. 2

Karger, S. 50

Karger, T. 50

King, R.F.G.J. 40

Kinjoh, K. 165

Koka, V. 107

Kosugi, T. 165

Kovacs, M.J. 131

Lafuente, P.J. 115

Larson, L. 59

Lasco, A. 127

Lazzari, M.A. 155

Lip, G.Y.H. 158

Livadiotaki, A. 2

Lund, E.D. 92

Lydataki, N. 2

McKenzie, M.E. 8

Mahé, I. 134

Makin, A.J. 158
Mallol, C. 194

Massberg, S. 16

Matsumoto, M. 33

Meij, M.A. de 76

Meister, A.F. 8

Melli, M. 44

Meschengieser, S.S. 155

Molostvov, G. 85

Moreno, A. 25

Morris, A. 85

Mühlbayer, D. 143

Mulder, C.J.J. 190

Müller, I. 16

Munkholm, P. 92

Nakamura, M. 165

Nederby Nielsen, J. 92

Nomura, T. 33

Nonaka, Y. 33

Nordström, H. 59

O'Connor, C.M. 8

Ohta, Y. 184

Ommen, G.V.A. van 195

Ortega, G. 25

Otten, J.M.M.B. 76

Papanikolaou, N. 2

Parma, B. 44

Paspatis, G.A. 2

Pawlak, R. 67

Peternel, P. 151

Pindur, G. 121

Porreca, E. 180

Potti, A. 80, 107

Ray, J.G. 51

Remkes, M.G.H. 111

Römisch, J. 143

Rose, P. 85

Rosing, J. 1

Rüdiger-von Hoch, S. 16

Ruggiero, M. 44

Ruiters, L. 196

Rullo, A. 180

Šabovič, M. 151

Sakata, K. 67

Sakurai, Y. 33

Salobir, B. 151

Sánchez de la Cuesta, F. 25
Sanchez Luceros, A.G. 155

Scamardi, R. 127

Schoor, C.A. van der 76

Schuster, A. 16

Schved, J.F. 194

Schwartz, J. 99

Seki, J. 184

Serebruany, V.L. 8

Sfyridaki, A. 2

Shibata, M. 33

Shmorgun, D. 51

Silverman, S.H. 158

Smorenburg, S.M. 76

Spitzer, S.G. 121

Stauss, H. 143

Stigendal, L. 59

Sue-Ling, H.M. 40

Sunagawa, M. 165

Suzuki, H. 33

Tadros, S. 107

Taka, T. 184

Takada, A. 67

Tate, G. 40

Triantafyllou, K. 2

Trifiletti, A. 127

Unanue, I. 115

Urano, T. 67

Vacas, M. 115

Vainer, J. 196

Vannucchi, S. 44

Vergnes, C. 194

Vermeulen, M. 111

Vries, R.A. de 190

Waller, L. 59

Wells, P.S. 131

Wiersma, T.G. 190

Yagi, H. 33

Yamamoto, J. 184

Yamamoto, Y. 33

Yegnanarayan, R. 80

Zierhut, W. 16

Zwaan, C. de 196

\section{KARGER}

(c) 2002 S. Karger AG, Base

Fax + 41613061234

E-Mail karger@karger.ch

www.karger.com
Accessible online at:

www. karger.com/pht 